\section{CEUS LI-RADS 2016 diagnostic scheme of the American College of Radiology released in September 2016}

The Liver Imaging Reporting and Data System ${ }^{\circledR}$ (LI-RADS $\left.{ }^{\circledR}\right)$ is an American College of Radiology (ACR) initiative whose immediate goal is to standardize reporting and data collection of non-invasive imaging for hepatocellular carcinoma (HCC). It provides the features for a confident diagnosis of HCC in the liver in at-risk patients and, for nodules not meeting HCC criteria, an ordinal classification based on the relative probability of HCC or non-hepatocellular malignancy. The Steering Committee is chaired by Prof Claude Sirlin (University of California, San Diego). The first LIRADS ${ }^{\circledR}$ scheme was released by the ACR website in 2011 (with subsequent updates in 2013 and 2014) and focuse on CT and MRI.

Contrast-Enhanced Ultrasound (CEUS) is recognized for its ability to characterise liver nodules in cirrhosis. In 2014, a decision was made to start work on an official
LI-RADS ${ }^{\circledR}$ scheme for CEUS. A Working Group was convened under the Chairmanship of Drs Yuko Kono (UCSD) and Andrej Lyshchik (Thomas Jefferson University, Philadelphia). They assembled a team of advisors which included American, Canadian and European experts. The Working Group, mainly meeting via regular telephone conferences, modified the existing LI-RADS® document to allow for the differences between contrast enhanced CT/MR and Ultrasound, especially the fact that ultrasound operates in real time and so is uniquely able to capture rapid and early haemodynamic events.

The Working Group's progress was reported at international meetings in 2015 and 2016 and the completed document was submitted to the LI-RADS $®$ Steering Committee body in April 2016; it was accepted by the Steering Committee in June 2016 and posted on the ACR website in Septem- ber 2016 where it is freely accessible: http://www.acr.org/quality-safety/resources/LIRADS

The publication of the ACR CEUS LIRADS ${ }^{\circledR}$ algorithm is likely to be of interest for many EFSUMB members performing abdominal ultrasound in patients with chronic liver disease. Importantly, CEUS LI-RADS® recognizes that HCC and nonspecific malignancies such as cholangiocarcinoma can be differentiated based on careful inspection of washout pattern: wash out that is "late" ( $\geq 60 \mathrm{~s})$ in onset and mild in degree is characteristic of HCC, while washout that is either "early" $(<60$ $\mathrm{sec}$ ) or marked in degree is nonspecific and raises concern for a non-HCC malignancy. Recognition by LI-RADS ${ }^{\circledR}$ that CEUS can differentiate HCC from non-HCC malignancy non-invasively is anticipated to build confidence in the use of CEUS for characterization of liver nodules in cirrhosis and restore its place in the few international hepatology guidelines that had removed it. Please visit the website, download the CEUS LI-RADS ${ }^{\circ}$ slides, pilot the system in your department, and feedback to the ACR committee.

\section{Yours sincerely}

Odd Helge Gilja, EFSUMB President,

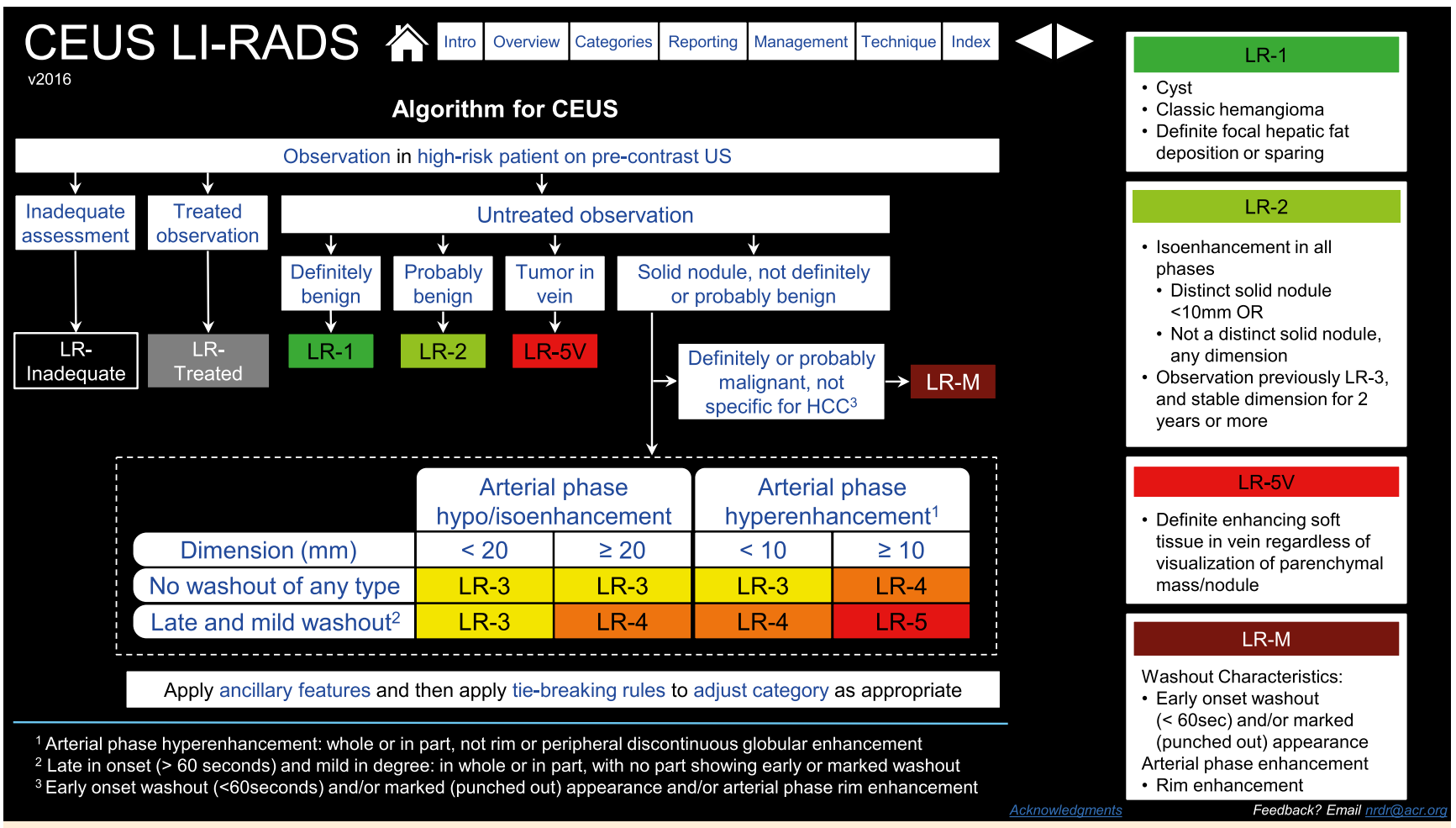

Fig.1 LIRADS algorithm for characterization of focal liver lesions (FLL) in the at-risk patient. (Courtesy of American College of Radiology) 